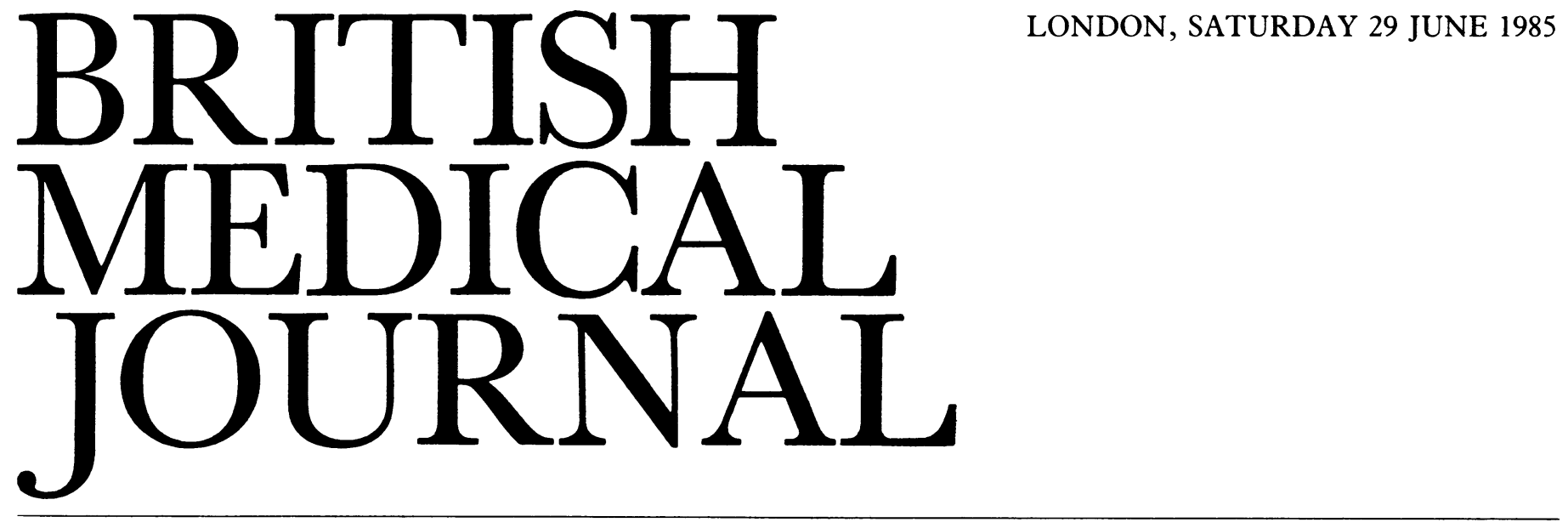

\title{
Urinary tract infection in children
}

Just as the clinical manifestations of urinary tract infection vary widely from birth to adolescence so do the nature and tempo of its treatment. In the newborn baby urinary tract infection may present simply as failure to thrive, as unexplained fever, or with increasing severity up to the fulminating "coliform" syndrome, which may include not only the urinary infection but also bacteraemia, meningitis, and jaundice (paradoxically often "obstructive"). Such severe infections must be treated immediately on the best guess principle with Escherichia coli as the most likely causal agent.' The results of culture with antimicrobial sensitivities are secondary information not available until the acute crisis is subsiding one way or the other. Some recent alarming data have shown that not only is urinary tract infection three times more common in the newborn cared for in the neonatal unit than in the home but also that the bacteria in the newborn in hospital belong to a strain specific to the neonatal unit, and different in $\mathrm{O}, \mathrm{K}$, and fimbrial antigens. ${ }^{2}$

Urinary tract infection continues to occur commonly after the neonatal period, but diagnosis is difficult during the first years of life. Unfortunately, this seems to be a critical time period in which vesicorenal reflux and severe damage may be caused to the still growing urological tract-with consequent permanent scarring of kidneys (reflux uropathy). If we are to prevent or to limit such damage from vesicorenal and intrarenal reflux and the consequent scarring we shall have to improve both the diagnosis and the treatment of urinary tract infection with proper follow up of potential relapse at least until school age. But how may urinary tract infections in babies and toddlers be identified, treated, and kept under control? There are no easy answers. Babies in nappies void urine frequently, smells of various types are common, and only an astute mother will associate crying on micturition with disease. Aspiration of urine from the bladder is an excellent and simple procedure for infants. Dipstream technique and quantitative dipslide culture have made accurate diagnosis much easier, avoiding the misleading growth of bacteria in liquid specimens kept at room temperature-which bedevils, especially, the results of urine culture from general practices. ${ }^{3}$

Acute urinary tract infection in toddlers may present with high fever, abdominal pain, convulsions, or other symptoms. This requires immediate treatment as soon as culture has been set up on a dipslide or the urine refrigerated. The best guess is that $E$ coli is responsible, and co-trimoxazole is probably the antimicrobial of choice. Investigations by ultrasound, "straight" $x$ ray (kidney, ureter, and bladder), investigation of the abdomen, intravenous urography, micturating cystic urethrography, and rarely cystoscopy have to be considered. For a hospital clinic a routine $x$ ray film plus ultrasound examination for first infections and intravenous urography for multiple or complex infections is a reasonable programme. In family practice there is a greater dilemma, since many more apparently first infections are seen, and these may not recur. The optimal investigation of first infection may be with ultrasound and radiography if facilities are available. If patients are selected for full investigation it should be concentrated on all children under 3 years, boys, and those with large bladders or kidneys, atypical organisms, haematuria, anaemia, hypertension, or nitrogen retention.

Acute urinary tract infection in preschool children usually presents with frequency, dysuria, urgency, fever, lumbar pain, haematuria, and return of enuresis in differing combinations. Overt urinary tract infection is recognised as needing prompt antimicrobial treatment. Less clear is the management of the perplexing group of children with latent urinary tract infection-on account of its frequency $(2.5 \%$ of school entrants ${ }^{4}$ ) and our lack of knowledge of the eventual outcome. Many girls with latent urinary tract infection have vesicoureteric reflux and cortical scars (reflux nephropathy). Most are asymptomatic, but others may have precipitancy and a return of enuresis. Probably in Britain 5000 girls annually enter school with latent urinary tract infection, and the size of the problem makes screening-let alone investigation and treatment-very expensive. Nevertheless, the clinician presented with a 5 year old girl with proved urinary tract infection, vesicorenal reflux, and renal scars understandably will tend to give immediate adequate antimicrobial treatment, followed perhaps by prophylactic long term treatment for two or more years in an effort to minimise or prevent subsequent damage to the growing kidneys.

Many girls treated by co-trimoxazole or nitrofurantoin long term remain relatively free of infection for long periods. ${ }^{5}$ The need for treatment in these circumstances was questioned after reports of trials which compared the results 
in treated and "control" groups. ${ }^{56}$ In some of these studies, however, the antimicrobial treatment was inadequate or intermittent or monitoring of compliance was inadequate: so not surprisingly little difference was found between the control and treated groups. These results have combined with the mistaken belief that further damage and scarring did not occur in schoolchildren to allow lackadaisical treatment. The authors of the excellent Newcastle study concluded that while continuous prophylactic antimicrobial treatment was highly effective in preventing and reducing urinary tract infection in treated girls as opposed to controls, screening of schoolchildren should be postponed until a non-radiological method could be devised to detect those with renal scarring. ${ }^{5}$ We now have this method-ultrasound. Should we screen now?

The paper from Smellie et al brings hard facts to bear on a previously speculative subject (p 1957). They studied the development of renal scars in 74 children (including some with latent urinary tract infection) who had 87 kidneys affected including 74 previously normal. Thirty four children were aged 5 years or more when scarring occurred. Diagnosis and treatment had been delayed in 45 , and in 58 symptomatic urinary tract infection occurred after a normal intravenous urogram and before scarring occurred. Vesicoureteric reflux was detected in $91 \%$ of these children. Interestingly, few scars, in this series, appeared in children aged over 7. Many paediatricians will see these results as confirming their view that both urinary tract infection and latent urinary tract infection should be treated.

The conclusions of the authors of the collaborative study are that early diagnosis, prompt effective treatment, investigation, and long term supervision of urinary infection are essential if renal scarring is to be reduced both in those with urinary tract infection aged $0-5$ years and also older children (p 1957). These are admirable sentiments, but the deed may prove harder to achieve than the wish. While the present study proves the occurrence of scarring it does not prove how to prevent it. The facts do not support the use of ureteric reimplantation as a method of preventing scars. Although no satisfactory controlled long term trial has yet been published of long term continuous prophylactic antimicrobial treatment (properly supervised, compliance ensured) to prove that scars are less likely and kidney growth more satisfactory than when infections are not prevented (or left untreated), the supposition is strongly in favour of treatment. Certainly leaving a child with urinary tract infection untreated, uninvestigated, and unsupervised in the future can no longer be justified-if it ever could. In recent years the fashion has been for shorter and shorter courses of antimicrobial treatment and for a rather slipshod attitude to following up infected babies and toddlers until middle school age. Smellie and her coworkers have effectively shattered complacency, and we must all look anew at this problem.

G C ARNEIL

Professor and Leonard Gow Lecturer,

Department of Child Health,

University of Glasgow,

Glasgow G3 8SJ

1 McAllister TA, Arneil GC. Choice of antimicrobial. In: Forfar JO, Arneil GC, eds. Textbook of paediatrics. Edinburgh: Churchill Livingstone, 1984:1868-70.
2 Tullus K, Horlin K, Svenson SB, Kallenius G. Epidemic outb

Tullus K, Horlin K, Svenson SB, Kallenius G. Epidemic outbreaks of pyelonephritis caused by nosocomial spread of P-fimbriated E coli in children. $\mathcal{Y}$ Infect Dis 1985;150:728-36 Mair MI. High incid
$1973 ; 13: 51-4$.

5 Newcastle Covert Bacteriuria Research Group. Covert bacteriuria in schoolgirls in Newcastleupon-Tyne: a S-year follow-up. Arch Dis Child 1981;56:585-92.

6 Cardiff-Oxford Bacteriuria Study Group. Sequelae of covert bacteriuria in schoolgirls. Lance $1978 ; 1: 889-93$

\section{Recurrent ventricular tachycardia}

Depending on the exact definition of sudden death there are between 50000 and 100000 victims in Britain each year and increasing numbers of potential victims are being resuscitated from sudden collapse outside hospital. Most of these episodes are not associated with myocardial infarction, ${ }^{2}$ though most patients have coronary artery disease. ${ }^{3}$

The exact mechanism of sudden death is not understood. Recently published information from fortuitously taped electrocardiographic recordings during sudden death has shown, however, that ventricular fibrillation is invariably preceded by a period of ventricular tachycardia. ${ }^{45}$ In two recent series of patients who had survived cardiac arrest outside hospital ("failed" sudden death) ventricular tachycardia could be induced in $61 \%$ of 153 patients, and ventricular fibrillation was rarely induced directly but was caused by degeneration of ventricular tachycardia. ${ }^{67}$ In patients who present with recurrent ventricular tachycardia the annual mortality from cardiac death is $30 \%$, and in most of these death is sudden. ${ }^{8}$

Ventricular tachycardia is now known also to be responsible for symptoms in clinical settings where until recently it had been almost unsuspected. For example, several studies have shown that ventricular tachycardia may be induced in half the patients with recurrent syncope of unknown origin; treatment based on the results of provocation testing has reduced the risk of recurrence..$^{910}$ The cause of syncope in patients with electrocardiographic evidence of intraventricular conduction disorders is generally assumed to be intermittent complete atrioventricular block. These patients, too, have a propensity to ventricular tachycardia, which could account for syncope. "

The correct diagnosis of ventricular tachycardia is achieved by painstaking inspection of the current electrocardiogram and detailed comparison with all previous recordings. A clear understanding of current diagnostic criteria is essential. ${ }^{12}$ The initial management strategy will depend on the clinical presentation. Intravenous infusion of an antiarrhythmic drug is appropriate if the tachycardia is haemodynamically stable, otherwise direct current shock is the method of choice. After successful termination of the tachycardia treatment must be given to prevent recurrences. In patients with coronary artery disease interventions designed to increase coronary blood flow or improve myocardial efficiency rarely affect the incidence of ventricular tachycardia, and long term antiarrhythmic treatment is usually required. When appropriate, the drug which has been effective intravenously may be given by mouth. Drugs of class I (such as disopyramide, tocainide, and flecainide) and class III (such as amiodarone and sotalol) are most effective for prevention of recurrences. At present, however, the efficacy of any chosen drug cannot be predicted and a therapeutic trial has to be made in every case. Empirical treatment with follow up on an outpatient basis is not only unreliable but potentially dangerous, especially if the patient has unstable tachycardias. Continuous electrocardiographic monitoring (tape or telemetry) with supervision in hospital is appropriate for patients with frequent episodes but is unsuitable if attacks are rare. Antiarrhythmic drugs may cause a reduction in the frequency of ventricular extrasystoles without suppressing sustained tachycardia or fibrillation ${ }^{13}$; when this effect has been shown by electrocardiographic 